\title{
The challenges of emotion recognition methods based on electroencephalogram signals: a literature review
}

\author{
I Made Agus Wirawan ${ }^{1,3}$, Retantyo Wardoyo ${ }^{2}$, Danang Lelono ${ }^{2}$ \\ ${ }^{1}$ Doctoral Program Department of Computer Science and Electronics, Faculty of Mathematics and Natural Science, Universitas \\ Gadjah Mada, Yogyakarta, Indonesia \\ ${ }^{2}$ Department of Computer Science and Electronics, Faculty of Mathematics and Natural Science, Universitas Gadjah Mada, \\ Yogyakarta, Indonesia \\ ${ }^{3}$ Education of Informatics Engineering Department, Faculty of Engineering and Vocational, Universitas Pendidikan Ganesha, \\ Bali, Indonesia
}

\begin{tabular}{l}
\hline \hline Article Info \\
\hline Article history: \\
Received May 25, 2021 \\
Revised Sep 16, 2021 \\
Accepted Oct 14, 2021 \\
\hline
\end{tabular}

Keywords:

Classification methods

Electroencephalogram

Emotion acquisition

Emotion elicitation

Emotion recognition

Feature extraction

Removing artifacts

\begin{abstract}
Electroencephalogram (EEG) signals in recognizing emotions have several advantages. Still, the success of this study, however, is strongly influenced by: i) the distribution of the data used, ii) consider of differences in participant characteristics, and iii) consider the characteristics of the EEG signals. In response to these issues, this study will examine three important points that affect the success of emotion recognition packaged in several research questions: i) What factors need to be considered to generate and distribute EEG data?, ii) How can EEG signals be generated with consideration of differences in participant characteristics?, and iii) How do EEG signals with characteristics exist among its features for emotion recognition? The results, therefore, indicate some important challenges to be studied further in EEG signals-based emotion recognition research. These include i) determine robust methods for imbalanced EEG signals data, ii) determine the appropriate smoothing method to eliminate disturbances on the baseline signals, iii) determine the best baseline reduction methods to reduce the differences in the characteristics of the participants on the EEG signals, iv) determine the robust architecture of the capsule network method to overcome the loss of knowledge information and apply it in more diverse data set.
\end{abstract}

This is an open access article under the $\underline{C C B Y-S A}$ license.

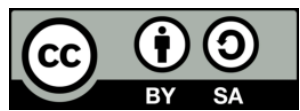

\section{Corresponding Author:}

Retantyo Wardoyo

Department of Computer Science and Electronics, Universitas Gadjah Mada

Building C, $4^{\text {th }}$ Floor, Sekip Utara, Bulaksumur, Senolowo, Sinduadi, Mlati District, Sleman Regency,

Yogyakarta Special Region 55281, Indonesia

Email: rw@ugm.ac.id

\section{INTRODUCTION}

Emotions are interactions and behaviors of human psychology which play an important role in everyday human social interactions. They usually arise as a response to certain conditions or problems representing a certain target to be achieved [1]. Positive emotions have the ability to maintain a person's mental state and increase work efficiency. In contrast, negative emotions cause mental state disorders and the buildup, at the top of the day, also leads to depression. Moreover, emotions arise spontaneously amid physical and physiological changes associated with human organs and tissues such as the brain, heart, skin, blood flow, muscles, facial expressions, and voice [2]. It is, therefore, important to acknowledge human emotions in an effort to understand human psychological interactions and behavior. There are generally two major categories of emotion recognition methods which are: i) physical or external aspects of humans and 
ii) physiological signals or internal aspects of humans. Meanwhile, the emotions expressed externally are usually deliberately hidden within the social environment [2]-[4]. These problems are mostly solved using the physiological signals from the central nervous system (CNS) via electroencephalogram (EEG) signals [5]. The EEG signals-based emotion recognition has several advantages such as: i) portability, low cost, and ease to line up [5], ii) rich spatial, temporal, and spectral data on human affective experiences which support the underlying neural mechanisms [6], [7], and iii) the occurrence of emotional reactions first in the human brain, especially within the subcortical, which means it is possible to directly reflect the changes in EEG signals in the human emotional condition [4], [6]. The EEG signals-supported emotion recognition studies have been widely applied within the 2 problem domains: medical and non-medical [8].

There has been rapid development of research on EEG signals-based recognition over the past five years in terms of data acquisition, data preprocessing, feature extraction, feature representation, and classification process [2], [7]-[11]. The success of this study, however, is strongly influenced by: i) distribution of the data used [9], ii) consider of differences in participant characteristics, such as personality traits, intellectual abilities, and gender in emotion reaction [12], [13], and iii) consider the characteristics of the EEG signals such as having a low frequency and containing spatial information on emotion recognition [14], [15]. In response to these issues, the research presented here will examine three important points that affect the success of emotion recognition packaged in several research questions: i) what factors need to be considered to generate and distribute EEG data to represent emotional reactions?, ii) how can EEG signals be generated with consideration of differences in participant characteristics?, and iii) how do EEG signals with characteristics exist among its features for emotion recognition? Therefore, the findings of this study are expected to be a reference for further research on emotion recognition based on EEG signals.

\section{RESEARCH METHOD}

This literature study was based on several articles retrieved from www.scopus.com, and the articles collected them through the two stages explained in the following subsections.

\subsection{Selection stage}

Several criteria based on the query were applied in the searching process in electronic databases, as shown in Figure 1. The retrieving process based on these queries results in 316 articles consisting of 171 conference papers and 145 journal articles.

\subsection{Analysis stage}

The next process is to analyze the articles obtained, and the process involved five stages: i) stage 1, focusing on the EEG signals and emotion recognition by checking the title and abstract, (ii) stage 2, checking the access of the articles, iii) stage 3, focusing on the three issues of the study by checking each article's introduction and methods, and iv) stage 4, Select the relevant article by checking the results and conclusion of each article. In Figure 2, the stages of selection and analysis of several articles are presented.

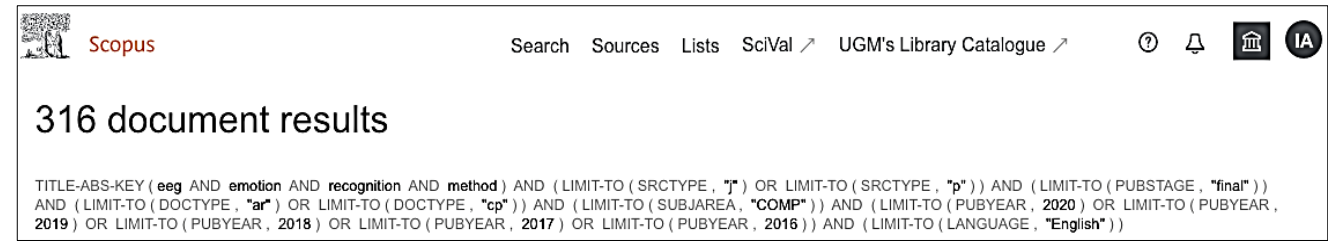

Figure 1. The query for searching articles

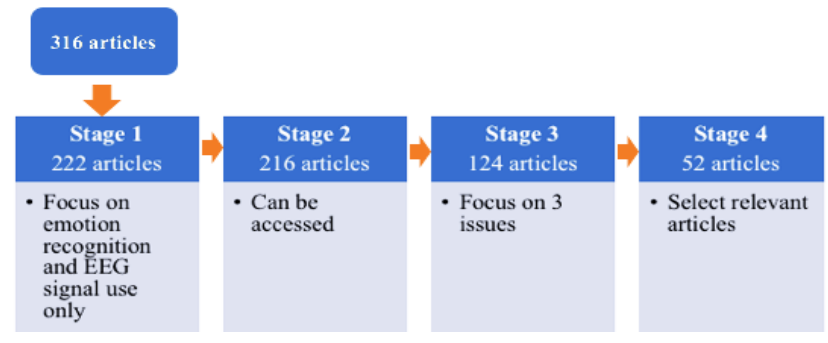

Figure 2. Selection and analysis stages of articles 
Based on the analysis stage, 52 relevant articles were obtained as references in this study. Apart from the 52 articles obtained from searching the Scopus database, this study also uses several additional articles totaling 37 articles to enrich the research study. So, this study uses 89 articles. The distribution of articles is based on three research questions (RQ) in this study are represented in Table 1. In distributing articles on each RQ, article redundancy can occur because each article answers more than one RQ.

Table 1. Analysis of the articles

\begin{tabular}{ccc}
\hline No & Research questions & Selected articles \\
\hline 1 & RQ 1 & 25 articles \\
2 & RQ 2 & 27 articles \\
3 & RQ 3 & 44 articles \\
& Total & 96 articles \\
\hline
\end{tabular}

\section{RESULTS AND DISCUSSION}

This study reviewed several issues associated with EEG signals-based emotional recognition, which can use for further research.

\subsection{RQ 1: What factors need to be considered to generate and distribute EEG data to represent emotional reactions?}

Several factors are taken into consideration in generating the EEG dataset for emotion recognition, including [11]:

a) Stimulus media: The literature studies showed several categories of stimuli to evoke emotions such as audio [16], [17], visual, and audio-visual media [14] as well as others including the ambient assisted living (AAL) technology [18], a combination of music, video, and game stimuli [19], mobile learning application [20], augmented reality (AR) [21], virtual reality (VR) [22], [23], and tactile enhanced multimedia (TEM) [24].

b) Proper stimuli presentation setup [11]. Several factors influencing the presentation of a stimulus, including the monitor screen size, lighting, viewing angles. Viewing distance and each of them is represented in the (1):

$$
\hat{y}=-14+70 x_{1}+2 x_{2}-0.0015 x_{2}^{2}+0.46 x_{3}^{2}
$$

where, $\hat{y}$ is the prediction of the preferred viewing distance (millimeters), $\mathrm{x}_{1}$ represents the TV monitor size (inches), $\mathrm{x}_{2}$ represents the illumination value in the room (lux), while $\mathrm{x}_{3}$ represents the viewing angle $\left(\right.$ degrees $\left.^{\circ}\right)$.

c) Standardization of experimental protocols [11]. The stimulus presentation in experimental design is an important factor influencing the type of emotion it evokes. Therefore, the general implementation protocol to extract emotions is explained in Figure 3.

\begin{tabular}{|c|c|c|c|c|}
\hline \multicolumn{2}{|c|}{$\begin{array}{c}\text { Pre-stimuli } \\
\text { (in minutes/seconds) }\end{array}$} & $\begin{array}{c}\text { Stimuli } \\
\text { (in minutes/seconds) }\end{array}$ & $\begin{array}{c}\text { Post-stimuli } \\
\text { (in minutes/seconds) }\end{array}$ \\
\hline R & C & W & S & M \\
\hline
\end{tabular}

Figure 3. Experimental design [11]

where, $R$ is a relaxation time or blank screen condition, $C$ is a countdown frame, $W$ is a white cross-presentation/baseline/normal state, $S$ is a presented stimulus, and $M$ is a self-assessment manikin $(\mathrm{SAM}) /$ rest time assessment.

Several studies have provided publicly available emotional datasets that other researchers can use, such as: DEAP [25], ASCERTAIN [26], GAMEEMO [27], DREAMER [28], MPED [29], SAFE [30], AMIGOS [31], MAHNOB-HCI [32], and SEED-IV [33]. Although some datasets are publicly available, these datasets may have an unbalanced distribution of data. The performance of the emotion recognition method depends on the data balance. Several studies that used public datasets such as DEAP were observed to have a high imbalance of emotional data. It was discovered that only respondents $16,28,30$, and 31 had 
balanced data for the arousal emotion label out of the 32 analyzed, while 10,14, 15, 22, and 32 had for emotional valence label [34]. There are several oversampling methods for data imbalance problems, such as adaptive synthetic sampling approach for imbalanced learning (ADASYN) [34], a novel fitness function, g-score genetic programming (GGP) [35], and synthetic minority oversampling technique (SMOTE) [36], [37]. However, most of the existing oversampling methods still found overlapping data in the final results, making it difficult to determine the decision limit for each class. The radius-SMOTE method can overcome this problem. This method emphasizes the initial selection approach by generating synthetic data based on the safe radius distance. However, the radius-SMOTE method has limitations in detecting noise in the data boundary area [38]. Therefore, the challenge for future studies is to determine robust methods for imbalanced EEG signals data.

\subsection{RQ 2: How can an EEG signal be generated with consideration of differences in participant characteristics?}

The participants' emotional reactions in EEG signals are strongly influenced by the different characteristics of participants, such as personality traits, intellectual abilities, and gender [9], [12]. The different characteristics of these participants can produce unique EEG signals patterns. Several studies have examined the use of baseline EEG signals to consider the different participant characteristics on experimental signals [39], [40]. It is important to note that the baseline EEG signals represent a calm state before a stimulus medium is given [25], [28], [31], [41], [42]. The steps of the baseline approach are cutting all the $\mathrm{C}$ channels in the baseline signals into several $\mathrm{N}$ segments with length $\mathrm{L}$, and each segment is averaged to obtain the BaseMean value using the (2). Furthermore, the baseline reduction process on the EEG test signal is carried out by subtracting the value of the EEG test signal from the baseline EEG signal value using the (3).

$$
\begin{aligned}
\text { BaseMean } & =\frac{\sum_{i=1}^{N} \text { BaseSignal }_{i}}{N} \\
\text { Clean_EEG } & =\text { Trial_EEG }_{j}-\text { BaseMean }
\end{aligned}
$$

The Clean_EEG $G_{j}$ signals are an EEG signal that represents a subjective emotional reaction according to a given media stimulus. Several baseline reduction methods are applicable to characterize signals data, such as the difference, relative difference, and fractional difference methods. However, they have been observed to be effective with only black tea aroma data [43]. However, the tea aroma has similar characteristics to the EEG signals data, such as containing a lot of noise and weak frequency intensity. Therefore, it is a challenge for future research to test the three baseline reduction methods suitable for use in EEG signals data.

The baseline signals approach has increased emotion recognition accuracy compared to without using the EEG baseline signals approach [40], [44], [45]. This approach also significantly increases the accuracy of recognizing 2 classes of emotions (arousal and valence) and 4 classes of emotions (high arousal positive valence; high arousal negative valence; low arousal negative valence; and low arousal positive valence) [46]. Other studies have also been proposed a correlation approach to determine the baseline signals that has a high correlation with the stimulus medium [47]. This approach can overcome cross-subject emotion recognition. Although the baseline EEG signals approach has produced high accuracy, this approach is strongly influenced by the quality of the baseline EEG signals [9]. Recording the baseline EEG signals that are free from external, internal, and disturbances originating from the participants' emotional reactions isn't easy to do even though the participants are in a calm state [39], [48], [49]. This disturbance causes the baseline EEG signals to be unable to characterize the differences in participant characteristics found in the EEG signals. There are several methods applicable to eliminate disturbance/artifacts in the EEG signals, including regression [50], wavelet transform [51], and blind source separation (BSS), which further include other techniques such as independent component analysis (ICA) usually applied for electrooculography (EOG) artifacts [5], [29] and eye blinking [52]. This ICA also has the ability to remove artifacts using statistical independence between EEG and artifacts [10]. Another method is the principal component analysis (PCA) used to analyze EEG intervals not contaminated artifacts by extracting eigenvalues and eigenvectors corresponding to the clean EEG signals [45]. Meanwhile, the signals mixed with the eye blink are usually decomposed into a series of intrinsic mode functions (IMFs) [2]. Most artifacts removal algorithms offer good performance, but this method only focuses on detecting and removing specific artifacts such as EOG, ECG, and EMG [50].

Another method that applies to eliminate external and internal interference on the EEG signals is the smoothing method [53], and the following are several smoothing methods, including mean filter, median 
filter, Savitzky-Golay filter, discrete Kalman filter, the Gaussian filter, and kernel density estimation kernel [54]. The process of smoothing the EEG data signals can smooth the fluctuations of the EEG signals and avoid the outlier EEG signals [55], [56]. Therefore, the next research challenge is determining the appropriate smoothing method to eliminate external or internal disturbances and emotional reactions in the baseline EEG signals and to study the best baseline reduction methods to consider the differences in the characteristics of the participants on the trial signals.

\subsection{How do EEG signals with characteristics exist among its features for emotion recognition?}

EEG signals have several important characteristics that need to be considered in emotion recognition, such as low frequency and spatial information. Several studies have identified some of these characteristics in feature extraction, feature representation, and the classification process [2], [7]-[11].

\subsubsection{Feature extraction}

This is usually used to obtain features relevant to the emotional state of the EEG signals, and the process is grouped into 3 as [8], [29]:

a) Time domain feature. This is based on the time domain of a signal, and some of it has been reviewed in previous studies, such as the mobility, complexity, and activity using Hjorth parameters [57], fractal dimension using the Higuchi method [58], [59], event-related potentials (ERP) features [60], and statistical feature [61].

b) Frequency domain feature. This is based on the frequency domain of a signal, and several features have been reviewed in previous studies such as power spectral density (PSD) [62], band power using wavelet transform [59], [63], mel-frequency cepstral coefficients (MFCCs) technique [64], and differential entropy (DE) [14], [15], [40], [65], [66].

c) Time-frequency domain feature. This is based on the time-frequency domain of a signal, and some examples reviewed in previous studies include short-time fourier transform (STFT) [67], discrete wavelet transform (DWT) Features [58], and Combination of statistical and fast Fourier transform (FFT) methods [68].

The differential entropy (DE) method has, however, been discovered to have the ability to distinguish high energy and low energy patterns from EEG frequencies [14] and also to characterize spatial information from EEG signals [15]. The features generated from the DE method are the most accurate and stable in emotion recognition compared to several others such as autoregressive parameters, fractal dimension, power spectral density (PSD), differential asymmetry (DASM), rational asymmetry (RASM), asymmetry (ASM), differential caudality (DCAU), wavelet features, and sample entropy [40], [65], [66]. The DE formula usually used to characterize an EEG signal is defined as (4) [40].

$$
h(X)=\int_{X}^{\infty} f(X) \log (f(x)) d x
$$

Where, $X$ is a random variable and $\mathrm{f}(\mathrm{x})$ is the probability density function of $X$. Meanwhile, the DE of the series $X$ obeying the Gauss distribution $\mathrm{N}\left(\mu, \delta^{2}\right)$ is expressed as $(5)$ :

$$
h(X)=\int_{-\infty}^{\infty} \frac{1}{\sqrt{2 \pi \delta^{2}}} e^{\frac{(x-\mu)^{2}}{2 \delta^{2}}} \log \left(\frac{1}{\sqrt{2 \pi \delta^{2}}} e^{-\frac{(x-\mu)^{2}}{2 \delta^{2}}}\right) d x \quad=\frac{1}{2} \log \left(2 \pi \delta^{2}\right)
$$

for a given frequency band $i$, the DE is defined as (6):

$$
h_{i}(\mathrm{X})=\frac{1}{2} \log \left(2 \pi e \delta^{2}\right)
$$

where, $e$ is Euler's constant (2.71828), $\delta^{2}$ the variance of the signal, and $h_{i}$ represents the DE of the corresponding EEG signals in the frequency band.

\subsubsection{Feature representation}

It is important to determine the appropriate method to represent the features of the EEG signals due to their spatial information characteristics. Some of the representation methods used in previous studies include the multiband feature matrix (MFM) [62], 2D mesh [69], maximal information coefficient (MIC) [70], and 3D cube [40]. The 3D Cube method can maintain spatial information between channels as well as frequency bands, including theta, alpha, beta, and gamma. It is based on the channel representation of the international system 10-20 mapped into a 9x9 matrix [40]. The 3D cube representation also inspires computer 
vision through three basic colors, including red, green, and blue (RGB). These RGB color channels have a value range of 0 to 255 which indicates the intensity of the color in each channel. The DE features are represented in a 3D cube, as indicated in the processes in Figure 4.

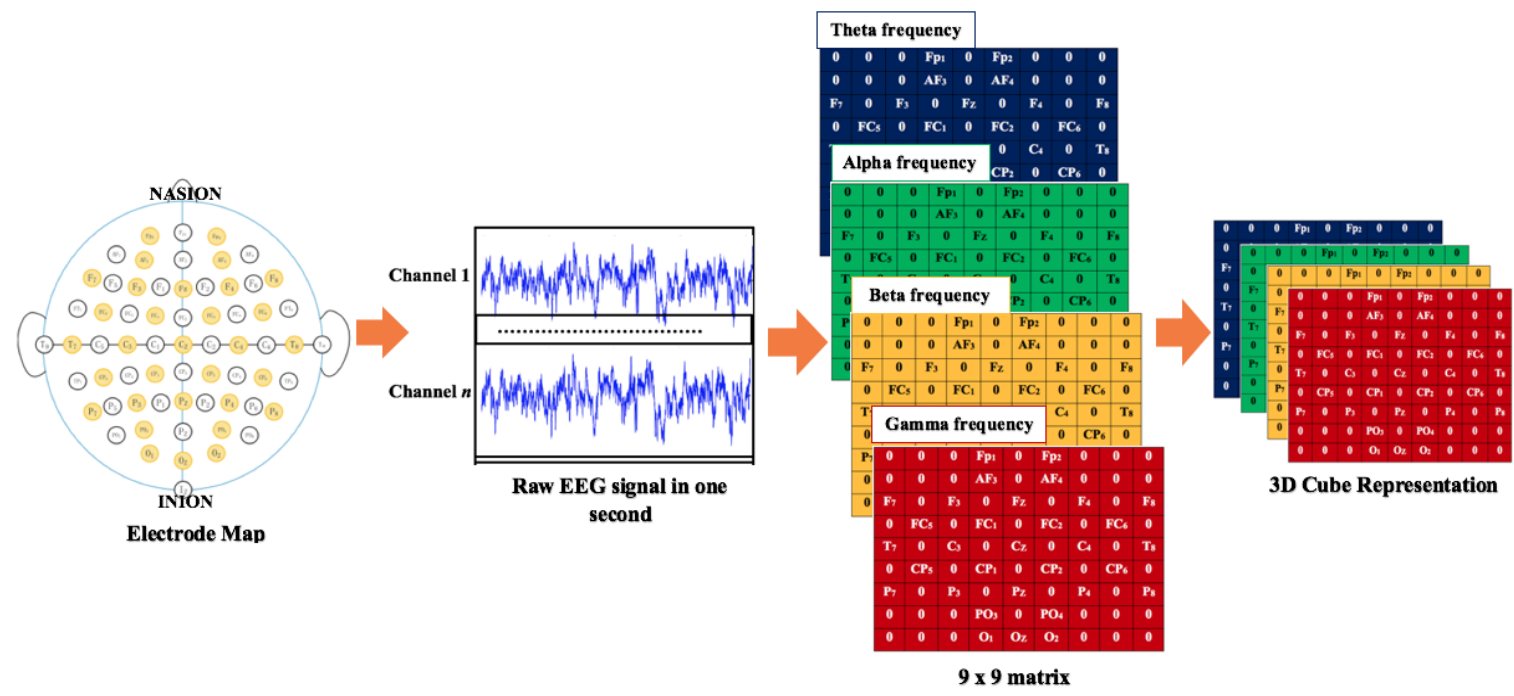

Figure 4. Feature representation based on 3D cube [40]

Based on Figure 4, every second, the EEG signals data generated from each EEG channel is decomposed into 4 frequency bands. Next, the feature extraction process is carried out for each frequency band. The feature value of each frequency band is then mapped into a 9x9 matrix so that it will produce 4 matrices. In the last stage, the 4 matrices are combined into a 3D cube [40]. The feature representation in the image is compared with the feature representation in the EEG signals in Table 2. The 3D cube-based feature representation has the ability to maintain spatial information between channels and also integrate between the frequency bands [40].

Table 2. Feature representation of images and EEG signals [40]

\begin{tabular}{|c|c|c|}
\hline & \multicolumn{2}{|c|}{ Domain } \\
\hline & Representation in computer vision & Representation in EEG signals \\
\hline Term & $\begin{array}{c}\text { Color image } \\
\text { Color channel (R, G, B) } \\
\text { Color Intensity }\end{array}$ & $\begin{array}{c}\text { EEG } 3 \text { cube } \\
\text { Frequency band }(\theta, \alpha, \beta, \gamma) \\
\text { Differential entrony feature }\end{array}$ \\
\hline
\end{tabular}

\subsubsection{Classification process}

The classification is the main process of emotion recognition important to be studied in addition to the feature extraction and representation processes. There are, however, two approaches to the classification of emotion through EEG and these include the machine learning and neural network approaches.

a) Machine learning approach: Some of the methods usually applied include decision tree (DT) [71], naïve bayes (NB) [72], quadratic discriminant analysis (QDA) [73], k-nearest neighbors (kNN) [58], [74], [75], linear discriminant analysis (LDA) [14], relevance vector machines (RVM) [67], xtreme gradient boosting (XGBoost) [76], support vector machine (SVM) [77]-[79], AdaBoost [80], logistic regression via variable splitting and augmented lagrangian (LORSAL) [81], random forest (RF) [56], [82], and graph regularized extreme learning machine (GELM) [83].

b) Neural network approach: This method include artificial neural network (ANN) [61], [63], [84] deep belief networks [70], [85], convolutional neural network (CNN) [40], [46], [86], [87], long short-term memory (LSTM) [66], generative adversarial networks (GAN) [88], capsule network (CapsNet) [45], [62], and hybrid methods [4], [44], [69].

Based on the articles obtained from the Scopus database from 2016-2020, CNN and SVM methods have been the most studied for emotion recognition based on EEG signals. In Figure 5, the distribution of several methods used for emotion recognition based on EEG signals is presented. Some deep learning 
methods, however, have superior accuracy compared to machine learning methods. The following summarizes the achievement of the highest accuracy of several deep learning methods in the classification of emotions based on EEG signals, as shown in Table 3.

Although the CNN method has slightly outperformed the capsule network method on the DREAMER dataset, however, the capsule network method has several advantages in recognizing emotions based on EEG signals, such as its ability to: (i) effectively characterize the spatial relationships between different features [89] and (ii) to be trained individually effective on a much smaller data scale compared to CNN [45]. Figure 6 shows the structure of the capsule network method generally consists of several parts, which include the following:

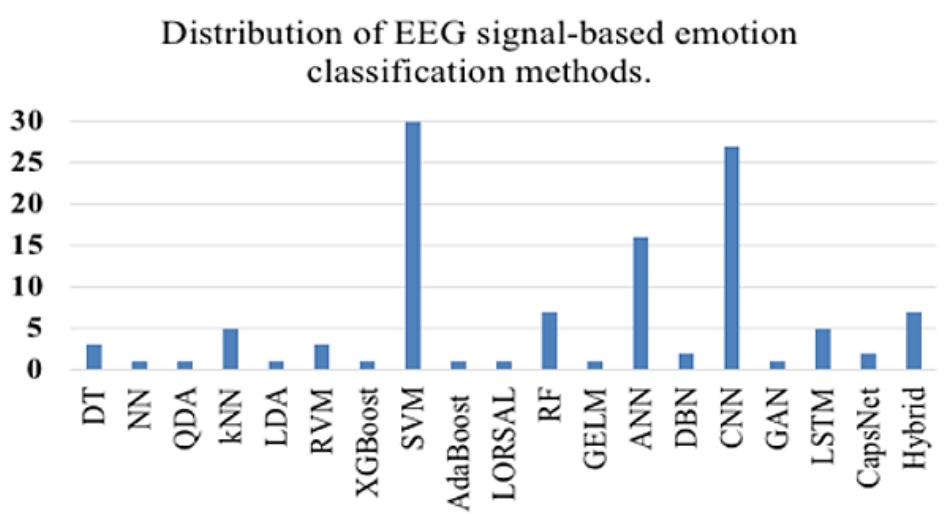

Figure 5. Distribution of EEG signals-based emotion classification method

Table 3. Comparison of the accuracy of deep learning methods

\begin{tabular}{|c|c|c|c|c|c|}
\hline No & Methods & Emotion classes & DEAP dataset & DREAMER dataset & AMIGOS dataset \\
\hline 1 & $\begin{array}{l}\text { MLF Capsule } \\
\text { Network [45] }\end{array}$ & 2 emotional classes & $\begin{array}{c}97.97 \% \text { for high/low } \\
\text { valence; } 98.31 \% \text { for } \\
\text { high/low arousal }\end{array}$ & $\begin{array}{l}94.59 \% \text { for high/low } \\
\text { valence; } \\
95.26 \% \text { for high/low } \\
\text { arousal }\end{array}$ & - \\
\hline 2 & RACNN [86] & 2 emotional classes & $\begin{array}{c}96.65 \% \text {; for high/low } \\
\text { valence; } 97.11 \% \text { for } \\
\text { high/low arousal }\end{array}$ & $\begin{array}{c}95.55 \% \text { for high/low } \\
\text { valence; } \\
97.01 \% \text { for high/low } \\
\text { arousal }\end{array}$ & - \\
\hline 3 & 3D-CNN [46] & 2 emotional classes & $\begin{array}{c}96.43 \% \text { for high/low } \\
\text { valence; } 96.61 \% \text { for } \\
\text { high/low arousal }\end{array}$ & - & $\begin{array}{c}96.96 \% \text { for high/low } \\
\text { valence; } \\
97.52 \% \text { for high/low } \\
\text { arousal }\end{array}$ \\
\hline 4 & 3D-CNN [46] & 4 emotional classes & $\begin{array}{l}\text { 93.53\% (high arousal } \\
\text { and positive valence; } \\
\text { high arousal and } \\
\text { negative valence; low } \\
\text { arousal and negative } \\
\text { valence; and low arousal } \\
\text { and positive valence) }\end{array}$ & - & $\begin{array}{l}\text { 95.95\% (high arousal and } \\
\text { positive valence; high } \\
\text { arousal and negative } \\
\text { valence; low arousal and } \\
\text { negative valence; and low } \\
\text { arousal and positive } \\
\text { valence) }\end{array}$ \\
\hline
\end{tabular}

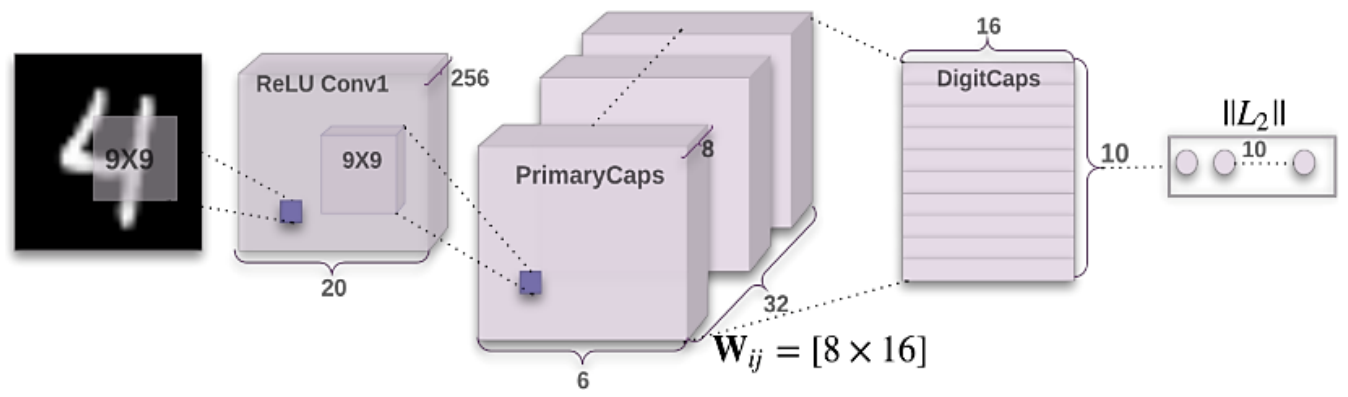

Figure 6. Capsule network architecture [89] 
a) Convolutional section where convolution process is conducted on the input data matrix using the ReLU activation function to produce the feature map to be used as the data input for the PrimaryCaps.

b) PrimaryCaps section consists of four processes, including: i) convolution, ii) concatenate, iii) bottleneck, and iv) reshape. The reshaping process, however, generates the vector data $u_{i}$, which represents the input vector value of the lower capsule i).

c) DigitCaps section includes several processes, including the following:

- The affine transformation process aims to represent the spatial relationship between the sub-objects of the total objects at a higher layer. This is further used to predict the correlation of these sub-objects with objects at higher levels. The vector $u_{i}$ and matrix $W_{i \mid j}$ are multiplied to produce vector $\hat{u}_{j \mid i}$, where $j$ represents the index of each class output.

$$
\hat{u}_{j \mid i}=W_{i \mid j} u_{i}
$$

- $\quad$ The weighted sum process was conducted based on the multiplication of the $C_{i j}$ with the input vector $\hat{u}_{j \mid i}$ to produce vector $S_{j}$.

$$
S_{j}=\sum_{i} C_{i j} \hat{u}_{j \mid i}
$$

The $C_{i j}$ is determined using a dynamic routing algorithm that iterates several times to generate $C_{i j}$ values (by default three times), as indicated in Table 4.

\begin{tabular}{|c|c|}
\hline \multicolumn{2}{|r|}{ Dynamic routing algorithm } \\
\hline 1: & procedure ROUTING $\left(\hat{u}_{j \mid \mathrm{i}}, r, l\right)$ \\
\hline 2: & for all capsule $i$ in layer $I$ and capsule $j$ in layer $(I+1): b_{i j} \leftarrow 0$ \\
\hline 3: & for $r$ iterations do \\
\hline 4: & for all capsule $i$ in layer $l: C_{i} \leftarrow$ SoftMax function $\left(b_{i j}\right)$ \\
\hline 5: & for all capsule $j$ in layer $(I+1): S_{j} \leftarrow \sum_{i} C_{i j} \hat{u}_{j \mid i}$ \\
\hline 6: & for all capsule $j$ in layer $(I+1): V_{j} \leftarrow \operatorname{squash}$ function $\left(S_{j}\right)$ \\
\hline 7: & for all capsule $i$ in layer $I$ and capsule $j$ in layer $(l+1): b_{i j} \leftarrow b_{i j}+\hat{u}_{j \mid i \cdot} \cdot V_{j}$ \\
\hline 8: & return $V_{j}$ \\
\hline
\end{tabular}

Table 4. Dynamic routing algorithm [89]

The process aims to project several predictive vectors $\left(\hat{u}_{j \mid i}\right)$ using the coupling coefficients $\left(C_{i j}\right)$ in order to produce the weighted sum value $\left(S_{j}\right)$.

- $\quad$ The squashing process generates an output vector $v_{j}$ for each class at a higher level $(l+1)$ and the maximum or highest value of $v_{j}$ determines the predicted class level. The squashing function to obtain the probability value in each prediction class is, therefore, represented using the Formula (9).

$$
V_{j}=\frac{\left\|S_{j}\right\|^{2}}{1+\left\|S_{j}\right\|^{2}} \frac{S_{j}}{\left\|S_{j}\right\|}
$$

d) The loss function calculation section was used to calculate the loss value based on the output and target values using the $\mathrm{L} 2$ regularization method.

$$
L_{e}=T_{e} \max \left(0, m^{+}-\left\|v_{e}\right\|\right)^{2}+\lambda\left(1-T_{e}\right) \max \left(0,\left\|v_{e}\right\|-m^{-}\right)^{2}
$$

$T_{e}$ is equal to 1 if the emotion class matches the target at $e, m^{-}=0.1$ and $m^{+}=0.9$, and is the down-weighting of the loss function. By default, $\lambda=0.5$, and $v_{e}$ represents the output vector of class $e$.

The capsule network method has several advantages over the others. Still, it allows the loss of knowledge information within the convolution process to work out feature maps and requires higher computation time than other deep learning methods [45]. This means the next research challenge is determining the acceptable architecture of the capsule network method to overcome the loss of knowledge information in the primary capsule. Moreover, it is also crucial to study the new architecture of the capsule network method to overcome the high computation time in the classification process. Considering that each emotion dataset has different characteristics, such as the number of channels used, the number of respondents, and the experimental strategy, for further research, it is also important to study the capsule 
network method in a more diverse data set and introduce 4 emotion classes. This study aims to obtain a more robust capsule network architecture on different datasets.

\section{CONCLUSION}

Although various studies have been conducted to overcome the three issues of emotion recognition based on EEG signals, there are several challenges to further study in the next research, include: i) determine robust methods for imbalanced EEG signals data, ii) determine the appropriate smoothing method to eliminate external or internal disturbances and emotional reactions in the baseline signals, iii) determine the best baseline reduction methods to consider the differences in the characteristics of the participants on the trial signals, and iv) determine the robust architecture of the capsule network method to overcome the loss of knowledge information and apply it in a more diverse data set. These challenges, in the future, are expected to produce robust models in emotion recognition based on EEG signals. This research study, however, has some limitations regarding the number of articles used. Therefore, further research needs to expand the scope of the emotion recognition domain, such as year, and topic.

\section{ACKNOWLEDGEMENTS}

The authors would like to acknowledge Universitas Gadjah Mada for providing facilities and funds through the Rekognisi Tugas Akhir research in 2021.

\section{REFERENCES}

[1] N. M. Krishna et al., "An efficient mixture model approach in brain-machine interface systems for extracting the psychological status of mentally impaired persons using EEG signals," IEEE Access, vol. 7, pp. 77905-77914, 2019, doi: 10.1109/ACCESS.2019.2922047.

[2] L. Shu et al., "A review of emotion recognition using physiological signals," Sensors (Switzerland), vol. 18, no. 7, 2018, doi: $10.3390 /$ s18072074

[3] P. Ekman, W. V Friesen, and R. C. Simons, "Is the startle reaction an emotion?," Journal of Personality and Social Psychology, vol. 49, no. 5, pp. 1416-1426, 1985, doi: 10.1037/0022-3514.49.5.1416.

[4] Y. Li, J. Huang, H. Zhou, and N. Zhong, "Human emotion recognition with electroencephalographic multidimensional features by hybrid deep neural networks," Applied Sciences, vol. 7, no. 10, Oct. 2017, Art. no. 1060, doi: 10.3390/app7101060.

[5] F. Al-Shargie, U. Tariq, M. Alex, H. Mir, and H. Al-Nashash, "Emotion recognition based on fusion of local cortical activations and dynamic functional networks connectivity: An EEG study," IEEE Access, vol. 7, pp. 143550-143562, 2019, doi: 10.1109/ACCESS.2019.2944008.

[6] T. Xu, Y. Zhou, Z. Wang, and Y. Peng, "Learning emotions EEG-based recognition and brain activity: A survey study on BCI for intelligent tutoring system," Procedia Computer Science, vol. 130, pp. 376-382, 2018, doi: 10.1016/j.procs.2018.04.056.

[7] X. Hu, J. Chen, F. Wang, and D. Zhang, "Ten challenges for EEG-based affective computing," Brain Science Advances, vol. 5, no. 1, pp. 1-20, Mar. 2019, doi: 10.1177/2096595819896200.

[8] A. Al-Nafjan, M. Hosny, Y. Al-Ohali, and A. Al-Wabil, "Review and classification of emotion recognition based on EEG braincomputer interface system research: A systematic review," Applied Sciences, vol. 7, no. 12, Art. no. 1239, Dec. 2017, doi: 10.3390/app7121239.

[9] J. Zhang, Z. Yin, P. Chen, and S. Nichele, "Emotion recognition using multi-modal data and machine learning techniques: A tutorial and review," Information Fusion, vol. 59, pp. 103-126, Jul. 2020, doi: 10.1016/j.inffus.2020.01.011.

[10] N. K. Bhandari and M. Jain, "Emotion recognition and classification using Eeg: A review," International Journal of Scientific and Technology Research, vol. 9, no. 2, pp. 1827-1836, 2020.

[11] P. Sarma and S. Barma, "Review on stimuli presentation for affect analysis based on EEG," IEEE Access, vol. 8, pp. 5199152009, 2020, doi: 10.1109/ACCESS.2020.2980893.

[12] C. M. Tyng, H. U. Amin, M. N. M. Saad, and A. S. Malik, "The influences of emotion on learning and memory," Frontiers in Psychology, vol. 8, Aug. 2017, doi: 10.3389/fpsyg.2017.01454.

[13] V. Kurbalija, M. Ivanović, M. Radovanović, Z. Geler, W. Dai, and W. Zhao, "Emotion perception and recognition: An exploration of cultural differences and similarities," Cognitive Systems Research, vol. 52, pp. 103-116, Dec. 2018, doi: 10.1016/j.cogsys.2018.06.009.

[14] E. S. Pane, A. D. Wibawa, and M. H. Pumomo, "Channel selection of EEG emotion recognition using stepwise discriminant analysis," in 2018 International Conference on Computer Engineering, Network and Intelligent Multimedia (CENIM), Nov. 2018, pp. 14-19, doi: 10.1109/CENIM.2018.8711196.

[15] D. W. Chen et al., "A feature extraction method based on differential entropy and linear discriminant analysis for emotion recognition," Sensors (Switzerland), vol. 19, no. 7, 2019, doi: 10.3390/s19071631.

[16] H. Bo, L. Ma, Q. Liu, R. Xu, and H. Li, "Music-evoked emotion recognition based on cognitive principles inspired EEG temporal and spectral features," International Journal of Machine Learning and Cybernetics, vol. 10, no. 9, pp. 2439-2448, Sep. 2019, doi: 10.1007/s13042-018-0880-z.

[17] S. A. Y. Al-Galal, I. F. T. Alshaikhli, A. W. B. A. Rahman, and M. A. Dzulkifli, "EEG-based emotion recognition while listening to Quran recitation compared with relaxing music using valence-arousal model," in Proceedings-2015 4th International Conference on Advanced Computer Science Applications and Technologies, ACSAT 2015, 2016, no. December, pp. 245-250, doi: 10.1109/ACSAT.2015.10.

[18] V. Meza-Kubo, A. L. Morán, I. Carrillo, G. Galindo, and E. García-Canseco, "Assessing the user experience of older adults using a neural network trained to recognize emotions from brain signals," Journal of Biomedical Informatics, vol. 62, pp. 202-209, Aug. 2016, doi: 10.1016/j.jbi.2016.07.004. 
[19] A. Anderson, T. Hsiao, and V. Metsis, "Classification of emotional arousal during multimedia exposure," in Proceedings of the 10th International Conference on PErvasive Technologies Related to Assistive Environments, Jun. 2017, pp. 181-184, doi: $10.1145 / 3056540.3064956$.

[20] M. Gasah, A. Baharum, N. Hidayah Mat Zain, S. Halamy, R. Hanapi, and N. Aizuddin Mat Noor, "Evaluation of positive emotion in children mobile learning application," Bulletin of Electrical Engineering and Informatics (BEEI), vol. 9, no. 2, pp. 818-826, Apr. 2020, doi: 10.11591/eei.v9i2.2073.

[21] F. Redzuan, A.-N. A. Khairuddin, and N. A. Daud, "Emotional augmented reality-based mobile learning design elements: a kansei engineering approach," Indonesian Journal of Electrical Engineering and Computer Science (IJEECS), vol. 14, no. 1, pp. 413-420, Apr. 2019, doi: 10.11591/ijeecs.v14.i1.pp413-420.

[22] T. Xu, R. Yin, L. Shu, and X. Xu, "Emotion recognition using frontal EEG in VR affective scenes," in IEEE MTT-S 2019 International Microwave Biomedical Conference, IMBioC 2019-Proceedings, 2019, vol. 1, pp. 1-4, doi: 10.1109/IMBIOC.2019.8777843.

[23] Y. Liu et al., "Human factors evaluation in maritime virtual simulators using mobile EEG-based neuroimaging," in Advances in Transdisciplinary Engineering, 2017, pp. 261-268.

[24] A. Raheel, S. M. Anwar, and M. Majid, "Emotion recognition in response to traditional and tactile enhanced multimedia using electroencephalography," Multimedia Tools and Applications, vol. 78, no. 10, pp. 13971-13985, May 2019, doi: 10.1007/s11042018-6907-3.

[25] S. Koelstra et al., "DEAP: A database for emotion analysis; using physiological signals," IEEE Transactions on Affective Computing, vol. 3, no. 1, pp. 18-31, 2012, doi: 10.1109/T-AFFC.2011.15.

[26] R. Subramanian, J. Wache, M. K. Abadi, R. L. Vieriu, S. Winkler, and N. Sebe, "ASCERTAIN: emotion and personality recognition using commercial sensors," IEEE Transactions on Affective Computing, vol. 9, no. 2, pp. 147-160, Apr. 2018, doi: 10.1109/TAFFC.2016.2625250.

[27] T. Burak, M. Gonen, and I. Turkoglu, "Biomedical signal processing and control database for an emotion recognition system based on EEG signals and various computer games-GAMEEMO §,” Biomedical Signal Processing and Control, vol. 60, 2020, Art. no. 101951, doi: 10.1016/j.bspc.2020.101951.

[28] S. Katsigiannis and N. Ramzan, "DREAMER: A database for emotion recognition through EEG and ECG signals from wireless low-cost off-the-shelf devices," IEEE Journal of Biomedical and Health Informatics, vol. 22, no. 1, pp. 98-107, 2018, doi: 10.1109/JBHI.2017.2688239.

[29] T. Song, W. Zheng, C. Lu, Y. Zong, X. Zhang, and Z. Cui, "MPED: A multi-modal physiological emotion database for discrete emotion recognition," IEEE Access, vol. 7, pp. 12177-12191, 2019, doi: 10.1109/ACCESS.2019.2891579.

[30] Z. Lan, Y. Liu, O. Sourina, L. Wang, R. Scherer, and G. Müller-Putz, "SAFE: an EEG dataset for stable affective feature selection," Advanced Engineering Informatics, vol. 44, Apr. 2020, Art. no. 101047, doi: 10.1016/j.aei.2020.101047.

[31] J. A. Miranda Correa, M. K. Abadi, N. Sebe, and I. Patras, "AMIGOS: A dataset for affect, personality and mood research on individuals and groups," IEEE Transactions on Affective Computing, no. i, pp. 1-14, 2018, doi: 10.1109/TAFFC.2018.2884461.

[32] M. Soleymani, J. Lichtenauer, T. Pun, and M. Pantic, "A multimodal database for affect recognition and implicit tagging," IEEE Transactions on Affective Computing, vol. 3, no. 1, pp. 42-55, 2012, doi: 10.1109/T-AFFC.2011.25.

[33] W. L. Zheng, W. Liu, Y. Lu, B. L. Lu, and A. Cichocki, "EmotionMeter: A Multimodal Framework for Recognizing Human Emotions," IEEE Transactions on Cybernetics, vol. 49, no. 3, pp. 1110-1122, 2019, doi: 10.1109/TCYB.2018.2797176.

[34] E. T. Pereira and H. Martins Gomes, "The role of data balancing for emotion classification using EEG signals," International Conference on Digital Signal Processing, DSP, vol. 0, pp. 555-559, 2016, doi: 10.1109/ICDSP.2016.7868619.

[35] D. Acharya, S. Goel, R. Asthana, and A. Bhardwaj, "A novel fitness function in genetic programming to handle unbalanced emotion recognition data," Pattern Recognition Letters, vol. 133, pp. 272-279, May 2020, doi: 10.1016/j.patrec.2020.03.005.

[36] R. Mohammed, J. Rawashdeh, and M. Abdullah, "Machine learning with oversampling and undersampling techniques: Overview study and experimental results," in 2020 11th International Conference on Information and Communication Systems (ICICS), Apr. 2020, pp. 243-248, doi: 10.1109/ICICS49469.2020.239556.

[37] G. Pradipta, R. Wardoyo, A. Musdholifah, and I. Sanjaya, "Improving classifiaction performance of fetal umbilical cord using combination of SMOTE method and multiclassifier voting in imbalanced data and small dataset," International Journal of Intelligent Engineering and Systems, vol. 13, no. 5, pp. 441-454, Oct. 2020, doi: 10.22266/ijies2020.1031.39.

[38] G. A. Pradipta, R. Wardoyo, A. Musdholifah, and I. N. H. Sanjaya, "Radius-SMOTE: A new oversampling technique of minority samples based on radius distance for learning from imbalanced data," IEEE Access, vol. 9, pp. 74763-74777, 2021, doi: 10.1109/ACCESS.2021.3080316.

[39] J. Cheng et al., "Emotion recognition from multi-channel EEG via deep forest," IEEE Journal of Biomedical and Health Informatics, vol. 2194, no. MAY, 2020, Art. no. 1, doi: 10.1109/jbhi.2020.2995767.

[40] Y. Yang, Q. Wu, Y. Fu, and X. Chen, "Continuous convolutional neural network with 3D input for EEG-based emotion recognition," in International Conference on Neural Information Processing, 2018, vol. 11307 LNCS, no. October 2018, pp. 433443, doi: 10.1007/978-3-030-04239-4_39.

[41] S. Narayana, R. R. V. Prasad, and K. Warmerdam, "Mind your thoughts: BCI using single EEG electrode," IET Cyber-Physical Systems: Theory and Applications, vol. 4, no. 2, pp. 164-172, 2019, doi: 10.1049/iet-cps.2018.5059.

[42] A. Dzedzickis, A. Kaklauskas, and V. Bucinskas, "Human emotion recognition: review of sensors and methods," Sensors, vol. 20, no. 3, Jan. 2020, Art. no. 592, doi: 10.3390/s20030592.

[43] D. Lelono, H. Nuradi, M. R. Satriyo, T. W. Widodo, A. Dharmawan, and J. E. Istiyanto, "Comparison of difference, relative and fractional methods for classification of the black tea based on electronic nose," in 2019 International Conference on Computer Engineering, Network, and Intelligent Multimedia (CENIM), Nov. 2019, pp. 1-7, doi: 10.1109/CENIM48368.2019.8973308.

[44] Y. Yang, Q. Wu, M. Qiu, Y. Wang, and X. Chen, "Emotion recognition from multi-channel EEG through parallel convolutional recurrent neural network," in 2018 International Joint Conference on Neural Networks (IJCNN), Jul. 2018, pp. 1-7, doi: 10.1109/IJCNN.2018.8489331.

[45] Y. Liu et al., "Multi-channel EEG-based emotion recognition via a multi-level features guided capsule network," Computers in Biology and Medicine, vol. 123, Aug. 2020, Art. no. 103927, doi: 10.1016/j.compbiomed.2020.103927.

[46] Y. Zhao, J. Yang, J. Lin, D. Yu, and X. Cao, "A 3D convolutional neural network for emotion recognition based on EEG signals," in 2020 International Joint Conference on Neural Networks (IJCNN), Jul. 2020, pp. 1-6, doi: 10.1109/IJCNN48605.2020.9207420.

[47] J. Xu, F. Ren, and Y. Bao, "EEG emotion classification based on baseline strategy," in Proceedings of 2018 5th IEEE International Conference on Cloud Computing and Intelligence Systems, CCIS 2018, 2019, pp. 43-46, doi: 10.1109/CCIS.2018.8691174.

[48] K. Gasper, L. A. Spencer, and D. Hu, "Does neutral affect exist? how challenging three beliefs about neutral affect can advance

The challenges of emotion recognition methods based on electroencephalogram ... (I Made Agus Wirawan) 
affective research," Frontiers in Psychology, vol. 10, Nov. 2019, doi: 10.3389/fpsyg.2019.02476.

[49] K. Gasper, "Utilizing neutral affective states in research: theory, assessment, and recommendations," Emotion Review, vol. 10, no. 3, pp. 255-266, Jul. 2018, doi: 10.1177/1754073918765660.

[50] X. Jiang, G. Bin Bian, and Z. Tian, "Removal of artifacts from EEG signals: A review," Sensors (Switzerland), vol. 19, no. 5. pp. 1-18, 2019, doi: 10.3390/s19050987.

[51] Y. M. Jin, Y. D. Luo, W. L. Zheng, and B. L. Lu, "EEG-based emotion recognition using domain adaptation network," in Proceedings of the 2017 International Conference on Orange Technologies, ICOT 2017, 2018, vol. 2018-Janua, pp. 222-225, doi: 10.1109/ICOT.2017.8336126.

[52] N. Thammasan, K. Moriyama, K. Fukui, and M. Numao, "Continuous music-emotion recognition based on electroencephalogram," IEICE Transactions on Information and Systems, vol. E99.D, no. 4, pp. 1234-1241, 2016, doi: 10.1587/transinf.2015EDP7251.

[53] A. Kawala-Sterniuk et al., "Comparison of smoothing filters in analysis of EEG data for the medical diagnostics purposes," Sensors, vol. 20, no. 3, Feb. 2020, Art. no. 807, doi: 10.3390/s20030807.

[54] P. Kowalski and R. Smyk, "Review and comparison of smoothing algorithms for one-dimensional data noise reduction," in 2018 International Interdisciplinary PhD Workshop (IIPhDW), May 2018, pp. 277-281, doi: 10.1109/IIPHDW.2018.8388373.

[55] X. Zhong, Z. Yin, and J. Zhang, "Cross-subject emotion recognition from EEG using convolutional neural networks," in 2020 39th Chinese Control Conference (CCC), Jul. 2020, pp. 7516-7521, doi: 10.23919/CCC50068.2020.9189559.

[56] G. K. P. Veeramallu, Y. Anupalli, S. kumar Jilumudi, and A. Bhattacharyya, "EEG based automatic emotion recognition using EMD and random forest classifier," in 2019 10th International Conference on Computing, Communication and Networking Technologies (ICCCNT), Jul. 2019, pp. 1-6, doi: 10.1109/ICCCNT45670.2019.8944903.

[57] T. Ergin, M. A. Ozdemir, and A. Akan, "Emotion recognition with multi-channel EEG signals using visual stimulus," TIPTEKNO 2019 - Tip Teknolojileri Kongresi, pp. 1-4, 2019, doi: 10.1109/TIPTEKNO.2019.8895242.

[58] A. M. Ismael, Ö. Alçin, K. H. Abdalla, and A. Şengür, "Two stepped majority voting for efficient EEG based emotion classification," Brain Informatics, no. July, 2020, doi: 10.21203/rs.3.rs-37469/v1.

[59] Z. Lan, O. Sourina, L. Wang, and Y. Liu, "Real-time EEG-based emotion monitoring using stable features," The Visual Computer, vol. 32, no. 3, pp. 347-358, Mar. 2016, doi: 10.1007/s00371-015-1183-y.

[60] M. Goyal, M. Singh, and M. Singh, "Classification of emotions based on ERP feature extraction," Proceedings on 2015 1st International Conference on Next Generation Computing Technologies, NGCT 2015, no. September, pp. 660-662, 2016, doi: 10.1109/NGCT.2015.7375203.

[61] M. Asadur Rahman, M. Foisal Hossain, M. Hossain, and R. Ahmmed, "Employing PCA and t-statistical approach for feature extraction and classification of emotion from multichannel EEG signal," Egyptian Informatics Journal, vol. 21, no. 1, pp. 23-35, Mar. 2020, doi: 10.1016/j.eij.2019.10.002.

[62] H. Chao, L. Dong, Y. Liu, and B. Lu, "Emotion recognition from multiband EEG signals using CapsNet," Sensors, vol. 19, no. 9, May 2019, Art. no. 2212, doi: 10.3390/s19092212.

[63] A. N. Nadira Mohammad Yosi, K. Azami Sidek, H. Sakti Yaacob, M. Othman, and A. Zamani Jusoh, "Emotion recognition using electroencephalogram signal," Indonesian Journal of Electrical Engineering and Computer Science (IJEECS), vol. 15, no. 2, pp. 786-793, Aug. 2019, doi: 10.11591/ijeecs.v15.i2.pp786-793.

[64] N. I. M. Razi, A. W. A. Rahman, and N. Kamarudin, "Detecting learning disabilities based on neuro-physiological interface of affect (NPIoA)," Indonesian Journal of Electrical Engineering and Computer Science (IJEECS), vol. 19, no. 1, pp. 163-170, 2020, doi: 10.11591/ijeecs.v19.i1.pp163-170.

[65] J. Li, Z. Zhang, and H. He, "Hierarchical convolutional neural networks for EEG-based emotion recognition," Cognitive Computation, vol. 10, no. 2, pp. 368-380, 2018, doi: 10.1007/s12559-017-9533-x.

[66] H. Jiang and J. Jia, "Research on EEG emotional recognition based on LSTM," in Bio-inspired Computing: Theories and Applications, 2019, pp. 409-417, doi: 10.1007/978-981-15-3415-7.

[67] Q. Gao, C. han Wang, Z. Wang, X. lin Song, E. zeng Dong, and Y. Song, "EEG based emotion recognition using fusion feature extraction method," Multimedia Tools and Applications, vol. 79, no. 37-38, pp. 27057-27074, 2020, doi: 10.1007/s11042-02009354-y.

[68] F. P. George, I. M. Shaikat, P. S. Ferdawoos Hossain, M. Z. Parvez, and J. Uddin, "Recognition of emotional states using EEG signals based on time-frequency analysis and SVM classifier," International Journal of Electrical and Computer Engineering (IJECE), vol. 9, no. 2, pp. 1012-1020, Apr. 2019, doi: 10.11591/ijece.v9i2.pp1012-1020.

[69] J. Chen, D. Jiang, Y. Zhang, and P. Zhang, "Emotion recognition from spatiotemporal EEG representations with hybrid convolutional recurrent neural networks via wearable multi-channel headset," Computer Communications, vol. 154, pp. 58-65, Mar. 2020, doi: 10.1016/j.comcom.2020.02.051.

[70] H. Chao, L. Dong, Y. Liu, and B. Lu, "Improved deep feature learning by synchronization measurements for multi-channel EEG emotion recognition," Complexity, vol. 2020, pp. 1-15, Mar. 2020, doi: 10.1155/2020/6816502.

[71] M. Mohamadi and A. M. Ekhtekhari Moghadam, "Improvement of EEG signal-based emotion recognition based on feature learning methods," in 2018 9th Conference on Artificial Intelligence and Robotics and 2nd Asia-Pacific International Symposium, Dec. 2018, pp. 14-18, doi: 10.1109/AIAR.2018.8769772.

[72] N. Y. Oktavia, A. D. Wibawa, E. S. Pane, and M. H. Purnomo, "Human emotion classification based on EEG signals using naïve bayes method," in 2019 International Seminar on Application for Technology of Information and Communication (iSemantic), Sep. 2019, pp. 319-324, doi: 10.1109/ISEMANTIC.2019.8884224.

[73] N. Sulthan, N. Mohan, K. A. Khan, S. S., and M. Shanir P.P, "Emotion recognition using brain signals," in 2018 International Conference on Intelligent Circuits and Systems (ICICS), Apr. 2018, pp. 315-319, doi: 10.1109/ICICS.2018.00071.

[74] A. Jalilifard, A. Rastegarnia, E. B. Pizzolato, and M. K. Islam, "Classification of emotions induced by horror and relaxing movies using single-channel EEG recordings," International Journal of Electrical and Computer Engineering (IJECE), vol. 10, no. 4, pp. 3826-3838, Aug. 2020, doi: 10.11591/ijece.v10i4.pp3826-3838.

[75] A. E. Putra, C. Atmaji, and F. Ghaleb, "EEG-based emotion classification using wavelet decomposition and K-nearest neighbor," in 2018 4th International Conference on Science and Technology (ICST), Aug. 2018, pp. 1-4, doi: 10.1109/ICSTC.2018.8528652.

[76] S. Parui, A. K. Roshan Bajiya, D. Samanta, and N. Chakravorty, "Emotion recognition from EEG signal using XGBoost algorithm," in 2019 IEEE 16th India Council International Conference (INDICON), Dec. 2019, pp. 1-4, doi: 10.1109/INDICON47234.2019.9028978.

[77] A. Patil and K. Behele, "Classification of human emotions using multiclass support vector machine," in 2017 International Conference on Computing, Communication, Control and Automation (ICCUBEA), Aug. 2017, pp. 1-4, doi: 
10.1109/ICCUBEA.2017.8463656.

[78] P. Ozel, A. Akan, and B. Yilmaz, "Synchrosqueezing transform based feature extraction from EEG signals for emotional state prediction," Biomedical Signal Processing and Control, vol. 52, pp. 152-161, Jul. 2019, doi: 10.1016/j.bspc.2019.04.023.

[79] M. Zangeneh Soroush, K. Maghooli, S. K. Setarehdan, and A. M. Nasrabadi, "Emotion recognition using EEG phase space dynamics and Poincare intersections," Biomedical Signal Processing and Control, vol. 59, May 2020, Art. no. 101918, doi: 10.1016/j.bspc.2020.101918.

[80] T. Lv, J. Yan, and H. Xu, "An EEG emotion recognition method based on AdaBoost classifier," in 2017 Chinese Automation Congress (CAC), Oct. 2017, pp. 6050-6054, doi: 10.1109/CAC.2017.8243867.

[81] C. Pan, C. Shi, H. Mu, J. Li, and X. Gao, "EEG-based emotion recognition using logistic regression with gaussian kernel and laplacian prior and investigation of critical frequency bands," Applied Sciences, vol. 10, no. 5, Feb. 2020, Art. no. 1619, doi: 10.3390/app10051619.

[82] E. S. Pane, A. D. Wibawa, and M. H. Purnomo, "Improving the accuracy of EEG emotion recognition by combining valence lateralization and ensemble learning with tuning parameters," Cognitive Processing, vol. 20, no. 4, pp. 405-417, Nov. 2019, doi: 10.1007/s10339-019-00924-Z.

[83] W.-L. Zheng, J.-Y. Zhu, and B.-L. Lu, "Identifying stable patterns over time for emotion recognition from EEG," IEEE Transactions on Affective Computing, pp. 1949-3045, 2016, doi: 10.1109/taffc.2017.2712143.

[84] P. Pandey and K. R. Seeja, "Subject independent emotion recognition from EEG using VMD and deep learning," Journal of King Saud University - Computer and Information Sciences, Nov. 2019, doi: 10.1016/j.jksuci.2019.11.003.

[85] N. Thammasan, K. Fukui, and M. Numao, "Application of deep belief networks in eeg-based dynamic music-emotion recognition," in 2016 International Joint Conference on Neural Networks (IJCNN), Jul. 2016, pp. 881-888, doi: 10.1109/JJCNN.2016.7727292.

[86] H. Cui, A. Liu, X. Zhang, X. Chen, K. Wang, and X. Chen, "EEG-based emotion recognition using an end-to-end regionalasymmetric convolutional neural network," Knowledge-Based Systems, vol. 205, Oct. 2020, Art. no. 106243, doi: 10.1016/j.knosys.2020.106243.

[87] T. Song, W. Zheng, P. Song, and Z. Cui, "EEG emotion recognition using dynamical graph convolutional neural networks," IEEE Transactions on Affective Computing, vol. 11, no. 3, pp. 532-541, Jul. 2020, doi: 10.1109/TAFFC.2018.2817622.

[88] Y. Dong and F. Ren, "Multi-reservoirs EEG signal feature sensing and recognition method based on generative adversarial networks," Computer Communications, vol. 164, pp. 177-184, Dec. 2020, doi: 10.1016/j.comcom.2020.10.004.

[89] S. Sabour, N. Frosst, and G. E. Hinton, "Dynamic routing between capsules," Computer Vision and Pattern Recognition, Oct. 2017.

\section{BIOGRAPHIES OF AUTHORS}

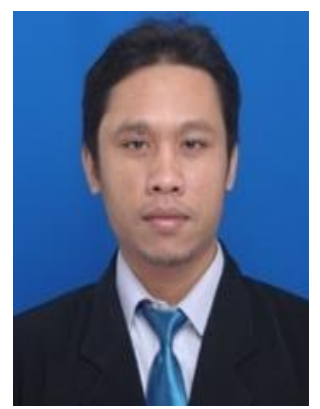

I Made Agus Wirawan (D) SC SC is currently pursuing his doctoral program in the Department of Computer Science and Electronics, Faculty of Mathematics and Natural Science, Universitas Gadjah Mada, Yogyakarta, Indonesia. He took her undergraduate (S.Kom) in Computer Science and Electronics Department, Faculty of Mathematics and Natural Science, Universitas Gadjah Mada, Yogyakarta and Master (M.Cs) in Computer Science and Electronics Department, Faculty of Mathematics and Natural Science, Universitas Gadjah Mada, Indonesia. His research areas of interest include machine learning, artificial intelligence, and e-learning. $\mathrm{He}$ can be contacted at email: imade.aguswirawan@mail.ugm.ac.id.

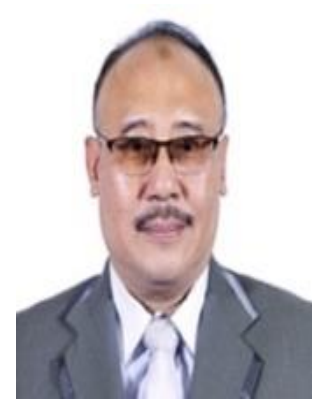

Retantyo Wardoyo (iD SC P is a lecturer and a researcher at the Department of Computer Science, Universitas Gadjah Mada. He obtained his bachelor's degree from Mathematics in Universitas Gadjah Mada, Indonesia. He obtained his master's degree in Computer Science at the University of Manchester, UK, and his doctoral degree from Computation at the University of Manchester Institute of Sciences and Technology, UK. His research interests include Intelligent Systems, Reasoning Systems, Expert Systems, Fuzzy Systems, Vision Systems, Group DSS and Clinical DSS, Medical Computing and Computational Intelligence. He can be contacted at email: rw@ugm.ac.id.



Danang Lelono (iD SC SC P is a lecturer and a researcher at the Department of Computer Science, Universitas Gadjah Mada. He obtained his bachelor's degree from Electronics and Instrumentation in Universitas Gadjah Mada, Indonesia. He obtained his master's degree from Electro/Technics in Universitas Gadjah Mada, Indonesia, and his doctoral degree from Computer Science in Universitas Gadjah Mada, Indonesia. His research interests include Smart Sensors, Intelligent Systems, Instrumentation and Control, Embedded Systems, ENose. He can be contacted at email: danang@ugm.ac.id. 